\title{
Impact of crystal orientation on ohmic contact resistance of enhancement-mode p-GaN gate high electron mobility transistors on $200 \mathrm{~mm}$ silicon substrates
}

\author{
Marleen Van Hove*, Niels Posthuma, Karen Geens, Dirk Wellekens, Xiangdong Li, and Stefaan Decoutere \\ imec, Kapeldreef 75, B-3001 Leuven, Belgium \\ *E-mail: Marleen.VanHove@imec.be \\ Received October 9, 2017; accepted November 16, 2017; published online February 14, 2018 \\ p-GaN gate enhancement mode power transistors were processed in a Si CMOS processing line on 200 mm Si(111) substrates using Au-free \\ metallization schemes. Si/Ti/Al/Ti/TiN ohmic contacts were formed after full recessing of the AIGaN barrier, followed by a HCl-based wet cleaning \\ step. The electrical performance of devices aligned to the [1120] and the perpendicular [1100] directions was compared. The ohmic contact

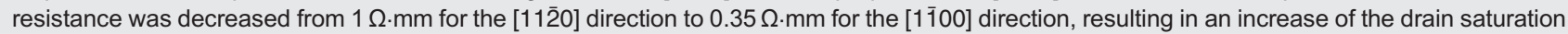 \\ current from 0.5 to $0.6 \mathrm{~A} / \mathrm{mm}$, and a reduction of the on-resistance from 6.4 to $5.1 \Omega \cdot \mathrm{mm}$. Moreover, wafer mapping of the device characteristics \\ over the $200 \mathrm{~mm}$ wafer showed a tighter statistical distribution for the [1100] direction. However, by using an optimized sulfuric/ammonia peroxide \\ (SPM/APM) cleaning step, the ohmic contact resistance could be lowered to $0.3 \Omega \cdot \mathrm{mm}$ for both perpendicular directions \\ (C) 2018 The Japan Society of Applied Physics
}

\section{Introduction}

Wide bandgap GaN-based power electronic devices are emerging as promising candidates for next generation highefficiency power converters owing to superior material properties such as high electric breakdown field, high electron saturation velocity, and high electron mobility in the $\mathrm{GaN}$ channel, formed at the $\mathrm{AlGaN} / \mathrm{GaN}$ heterojunction. ${ }^{1-3)}$ However, to replace commercially available $\mathrm{Si}$ power devices, the GaN devices should be enhancementmode (e-mode), and fabricated by a low-cost, reproducible and reliable production process. While e-mode operation can be readily achieved by adding a p-type doped $\mathrm{p}-\mathrm{AlGaN}$ or $\mathrm{p}-\mathrm{GaN}$ layer under the gate, hereby lifting the conduction band at equilibrium and resulting in electron depletion, ${ }^{4-7)}$ the ability to manufacture $\mathrm{GaN}$-on-Si power devices in existing $200 \mathrm{~mm}$ production facilities offers further cost competitiveness to the Si power technology. ${ }^{8-14)}$

An important hurdle for the realization of cost-effective GaN-on-Si devices, is however the realization of reproducible and reliable source and drain contacts with low ohmic contact resistance. To make $\mathrm{GaN}$ devices fully compatible with Si CMOS technology, Au-free ohmic contacts are necessary, both to lower the cost and the risk of crosscontamination with Si devices. Moreover, a low alloy temperature is preferred because this typically results in a smoother surface morphology, necessary for compatibility with CMOS stepper lithography alignment requirements.

As was demonstrated by Malmros et al., ${ }^{15}$ ) low contact resistance $R_{\mathrm{c}}$ and low alloy temperature for $\mathrm{Au}$-free schemes can be achieved by replacing the commonly used Ti/Albased ohmic metal stack by a Ta/Al-based stack resulting in $R_{\mathrm{c}}$ as low as $0.06 \Omega \cdot \mathrm{mm}$ for an alloy temperature of $550{ }^{\circ} \mathrm{C}$. $\mathrm{Ta} /$ Al-based ohmic contacts are however not directly compatible with CMOS processing lines, because Ta is only available in aggressively scaled submicron CMOS technologies typically on a shared $\mathrm{Ta} / \mathrm{Cu}$ metallization platform. For this reason, preferred metals for Au-free CMOS-compatible ohmic contact formation on $\mathrm{AlGaN} / \mathrm{GaN}$ high electron mobility transistors (HEMTs) are the commonly used interconnect metals in the older CMOS technology nodes: Ti, $\mathrm{Al}, \mathrm{TiN}$ and $\mathrm{W}$. We have previously shown that a $\mathrm{Ti} / \mathrm{Al} / \mathrm{W}$
$(20 / 100 / 20 \mathrm{~nm})$ ohmic metal stack, alloyed at $600^{\circ} \mathrm{C}$, results in ohmic contacts with relatively low $R_{\mathrm{c}}(0.65 \Omega \cdot \mathrm{mm}) .{ }^{16)}$

The mechanism for Au-free ohmic contact formation is still under debate. ${ }^{17,18)}$ The importance of the Ti/Al ratio in determining the optimum alloy temperature for $\mathrm{Ti} / \mathrm{Al} / \mathrm{W}$ contacts was recently discussed by Constant et al. ${ }^{19)}$ A very different optimal alloy temperature has been observed for Tiand Al-rich ohmic metal stacks. Ti-rich ohmic metal stacks (Ti quantity higher than 25 at. \%) typically require a high alloy temperature while, in agreement with our observations, the optimum alloy temperature is typically in the $500-600^{\circ} \mathrm{C}$ range for Al-rich ohmic metal stacks. ${ }^{16)}$

For both Au-containing ${ }^{20-24)}$ and Au-free ohmic contacts, ${ }^{25-31)}$ it was shown that the ohmic contact resistance can be further improved by partial or complete removal of the $\mathrm{AlGaN}$ barrier. Indeed, because of the insulating nature of the $\mathrm{AlGaN}$ barrier, ohmic contact formation relies partly on carrier tunneling across this barrier, and partial or complete removal of the $\mathrm{AlGaN}$ barrier was shown to facilitate ohmic contact formation. Complete removal of the AlGaN barrier has the advantage of a wider process window but, because the ohmic contact is formed by direct contact of the ohmic metal with the two-dimensional electron gas (2DEG) at the recess sidewall, control of the etch damage, the sidewall profile and optimization of the wet cleaning before ohmic metal deposition is of utmost importance, especially for Aufree ohmic contacts alloyed at low temperature because the metal in-diffusion is limited.

We have previously reported that by using complete AlGaN barrier recess etching, and a $\mathrm{HCl}$-based wet cleaning step prior to deposition of an Al-rich $\mathrm{Ti} / \mathrm{Al} / \mathrm{TiN}$ metal stack and alloy at $550{ }^{\circ} \mathrm{C}, R_{\mathrm{c}}$ can be lowered to $0.62 \Omega \cdot \mathrm{mm} \cdot{ }^{26,27)} \mathrm{In}$ this paper we report on further ohmic contact optimization specifically for $\mathrm{p}-\mathrm{GaN}$ gate enhancement mode HEMTs. By rotating the transistor to a different crystal orientation, hereby exposing a different crystal plane at the ohmic recess sidewall, the possible impact of the ohmic recess profile and wet chemical cleaning efficiency on the ohmic contact resistance can be studied.

\section{Experimental methods}

Most GaN-on-Si lateral power devices are fabricated in Ga- 


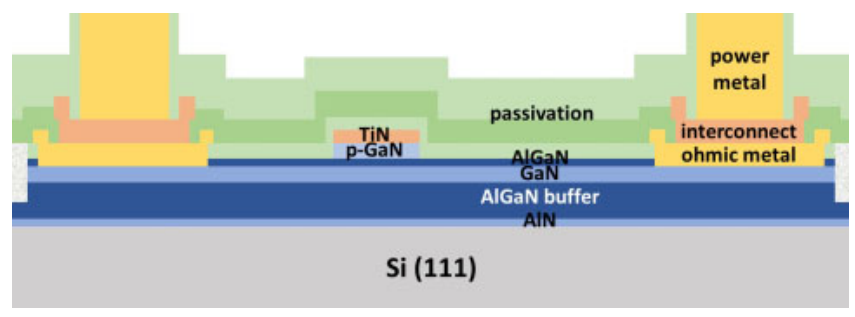

Fig. 1. (Color online) Schematic of $\mathrm{p}-\mathrm{GaN}$ gate enhancement-mode device.

face GaN crystals, and the electron transport in the 2DEG, that is formed at the $\mathrm{AlGaN} / \mathrm{GaN}$ heterojunction, is in the polar $c$-plane of the wurtzite $\mathrm{GaN}$ crystal. Because of the symmetry of the wurtzite crystal, the electron transport in $c$ plane HEMTs was shown to be isotropic, both theoretically and experimentally. ${ }^{32,33)}$ When the GaN crystal is however patterned with dry etching during the transistor fabrication, non-equivalent non-polar crystal planes, orthogonal to the polar $c$-plane, are exposed.

The fabrication of the e-mode $\mathrm{p}-\mathrm{GaN}$ gate HEMTs on $200 \mathrm{~mm}$ GaN-on-Si wafers was performed in a Si CMOS processing line, requiring Au-free and tool-compatible process steps (Fig. 1). The growth of the III-N layers was carried out by metalorganic chemical vapor deposition (MOCVD). The $\mathrm{p}-\mathrm{GaN}$ gate layer was grown in sequence with the (Al)GaN buffer, $\mathrm{GaN}$ channel and $\sim 13 \mathrm{~nm}$-thick $\mathrm{Al}_{0.25} \mathrm{Ga}_{0.75} \mathrm{~N}$ barrier layer. ${ }^{34)}$ The buffer thickness was $3.2 \mu \mathrm{m}$ and was optimized for $200 \mathrm{~V}$ applications.

After growth of the epitaxial stack, the gate was defined by the stacked gate patterning approach, etching the TiN gate metal and p-GaN layer in one sequence. A selective $\mathrm{p}-\mathrm{GaN}$ to $\mathrm{AlGaN}$ dry etch step was applied using a $\mathrm{BCl}_{3} / \mathrm{SF}_{6}$ chemistry. A $\mathrm{SiO}_{2}$ passivation layer was deposited on the $\mathrm{AlGaN}$ layer in the access area. The recessed ohmic contacts for source and drain were formed by dry etching of the passivating $\mathrm{SiO}_{2}$ layer in $\mathrm{CF}_{4}$ and the complete AlGaN barrier layer down to the $\mathrm{GaN}$ channel in $\mathrm{BCl}_{3}$, followed by the resist strip in an $\mathrm{O}_{2}$ plasma and a wet EKC265 polymer removal step. A HCl-based clean was carried out prior to the $\mathrm{Si} / \mathrm{Ti} / \mathrm{Al} / \mathrm{Ti} / \mathrm{TiN}(5 / 5 / 100 / 20 / 60 \mathrm{~nm})$ ohmic metal deposition and ohmic alloy at $565^{\circ} \mathrm{C}$. The process flow was completed by back-end metallization and passivation modules. The addition of a Si-containing layer in the ohmic metal stack was shown to result in a lower $R_{\mathrm{c}}$ by the formation of interfacial $\mathrm{Ti}_{x} \mathrm{Si}_{y}$ alloys. ${ }^{35,36)}$

As indicated in Fig. 2, the gates were oriented along the

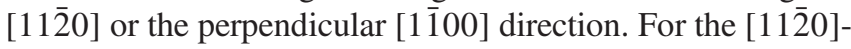
oriented transistors the ohmic $\mathrm{AlGaN}$ recess exposes the non-polar (1100) $m$-plane at the etched sidewalls, while the non-polar (1120) $a$-plane is exposed for the [1 100$]$-rotated transistors. The devices, optimized for $200 \mathrm{~V}$ applications, have a gate width $W_{\mathrm{G}}$ of $100 \mu \mathrm{m}$, a gate length $L_{\mathrm{G}}$ of $1 \mu \mathrm{m}$, a gate-source distance $L_{\mathrm{SG}}$ of $0.75 \mu \mathrm{m}$, and a gate-drain distance $L_{\mathrm{GD}}$ of $6 \mu \mathrm{m}$.

\section{Results and discussion}

The 2DEG sheet resistance $R_{\mathrm{sh}}$ of the AlGaN/GaN HEMT, which is affected by $\mathrm{Mg}$-in-diffusion from the $\mathrm{p}-\mathrm{GaN}$ epitaxial growth and $\sim 1 \mathrm{~nm} \mathrm{AlGaN}$ thickness loss in the selective $\mathrm{p}-\mathrm{GaN}$ dry etch step, ${ }^{34)}$ was mapped over the full

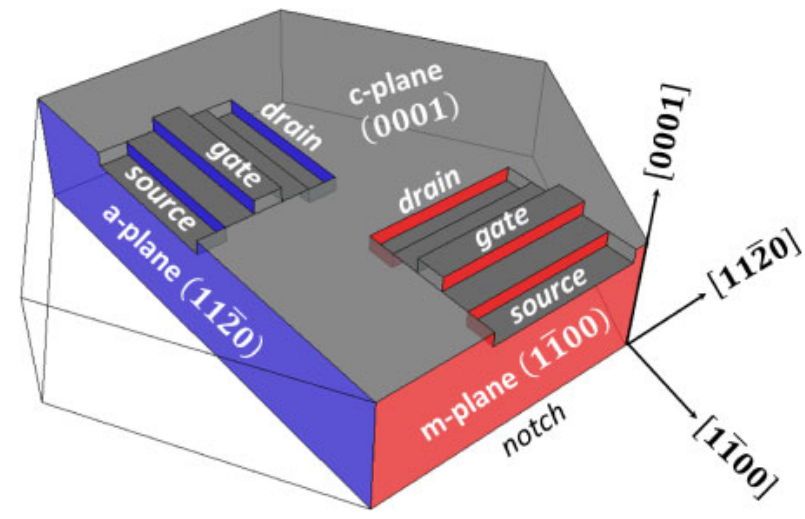

Fig. 2. (Color online) Schematic drawing of the transistor orientations parallel to the $[11 \overline{2} 0]$ direction or parallel to the [1 $\overline{1} 00]$ direction.
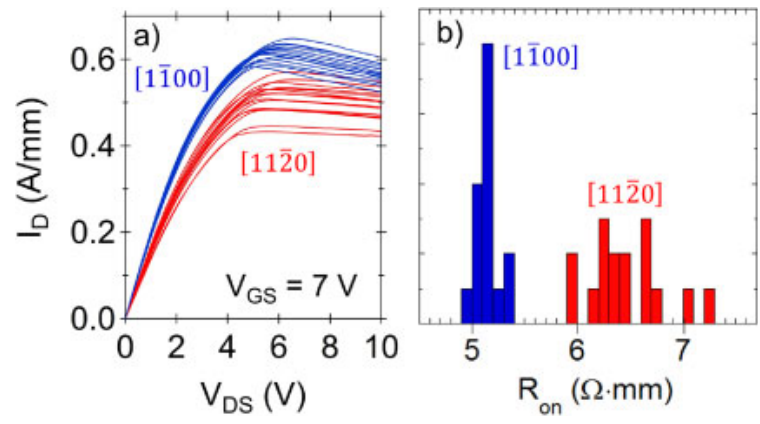

Fig. 3. (Color online) (a) $I_{\mathrm{D}}-V_{\mathrm{DS}}$ characteristics and (b) histogram of $R_{\mathrm{on}}$ for transistors parallel to the [11 20$]$ direction or parallel to the [1 100$]$ direction for the $\mathrm{HCl}$-based wet cleaning before ohmic metal deposition.

$200 \mathrm{~mm}$ wafer by van der Pauw measurements. The median $R_{\text {sh }}$ value and standard deviation over the full $200 \mathrm{~mm}$ wafer are $525 \pm 20 \Omega /$ sq. The contact resistance however, measured by the transfer length method (TLM), resulted in a contact resistance $R_{\mathrm{c}}$ of $1.0 \pm 0.2 \Omega \cdot \mathrm{mm}$ for the transistors aligned along the [112̄0] direction, while $R_{\mathrm{c}}$ was as low as $0.35 \pm 0.05 \Omega \cdot \mathrm{mm}$, with a tighter statistical distribution, along the perpendicular [1 $\overline{1} 00]$ direction. ${ }^{37)}$ This remarkable difference in contact resistance is reflected in a clearly higher drain current and a lower transistor on-resistance $\left(R_{\mathrm{on}}\right)$ with a tighter distribution for the [1100] transistors (Fig. 3). The $R_{\text {on }}$ values are $5.15 \pm 0.10 \Omega \cdot \mathrm{mm}$ for the transistors along the

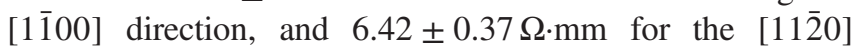
direction. Also, the transfer characteristics for both transistor orientations were mapped, and no difference was found in threshold voltage $V_{\text {th }}$ nor drain and gate leakage currents (Fig. 4). Moreover, the device breakdown voltage and dispersion were unaffected by the transistor rotation. The breakdown voltage, defined at $1 \mu \mathrm{A} / \mathrm{mm}$ drain leakage, was $\sim 535 \mathrm{~V}$ and the device dispersion was below $20 \%$ at $200 \mathrm{~V}$ for both transistor orientations (not shown).

A first possible mechanism to explain the difference in ohmic contact resistance between both crystal orientations is a different ohmic recess profile after the $\mathrm{BCl}_{3}$ dry ohmic recess etch step, though reports on preferential crystallographic dry etching in GaN are rare. ${ }^{38)}$ For a more sloped ohmic recess profile, the 2DEG-depleted region is expected to be wider and hence there is a higher resistance to inject electrons from the ohmic metal. For this reason, a higher 

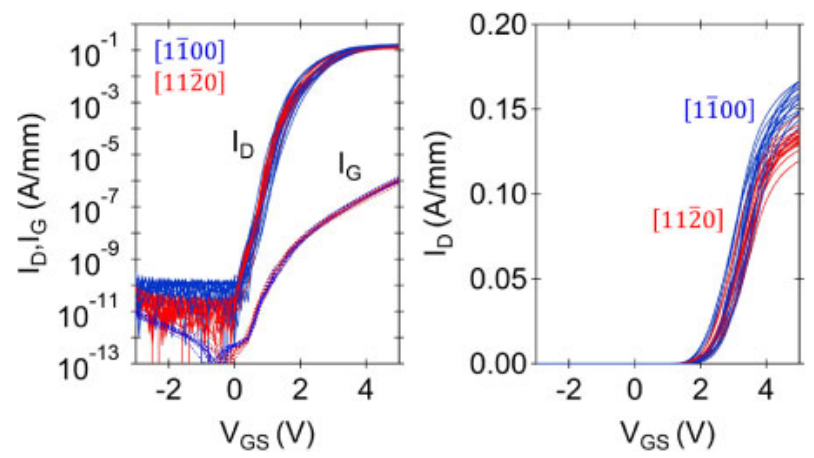

Fig. 4. (Color online) Comparison of $I_{\mathrm{D}}-V_{\mathrm{GS}}$ and $I_{\mathrm{G}}-V_{\mathrm{GS}}$ characteristics $\left(V_{\mathrm{DS}}=1 \mathrm{~V}\right)$ for $\mathrm{p}-\mathrm{GaN}$ gate e-mode transistors oriented parallel to the [11 20$]$ direction or parallel to the [1100] direction.
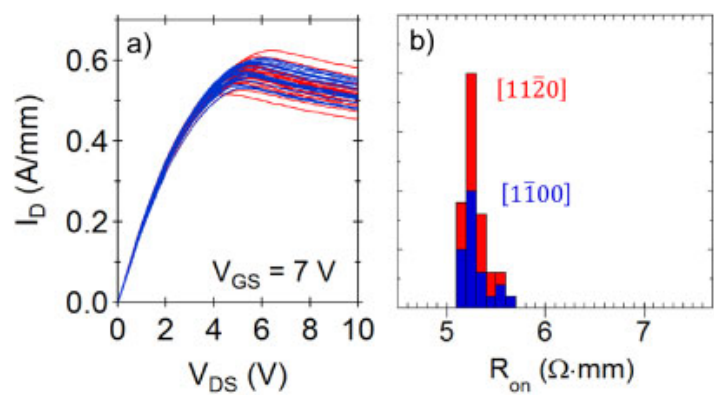

Fig. 6. (Color online) (a) $I_{\mathrm{D}}-V_{\mathrm{DS}}$ characteristics and (b) histogram of $R_{\mathrm{On}}$ for transistors parallel to the [1120] direction or parallel to the [1 100$]$ direction for the SPM/APM-based wet cleaning before ohmic metal deposition.
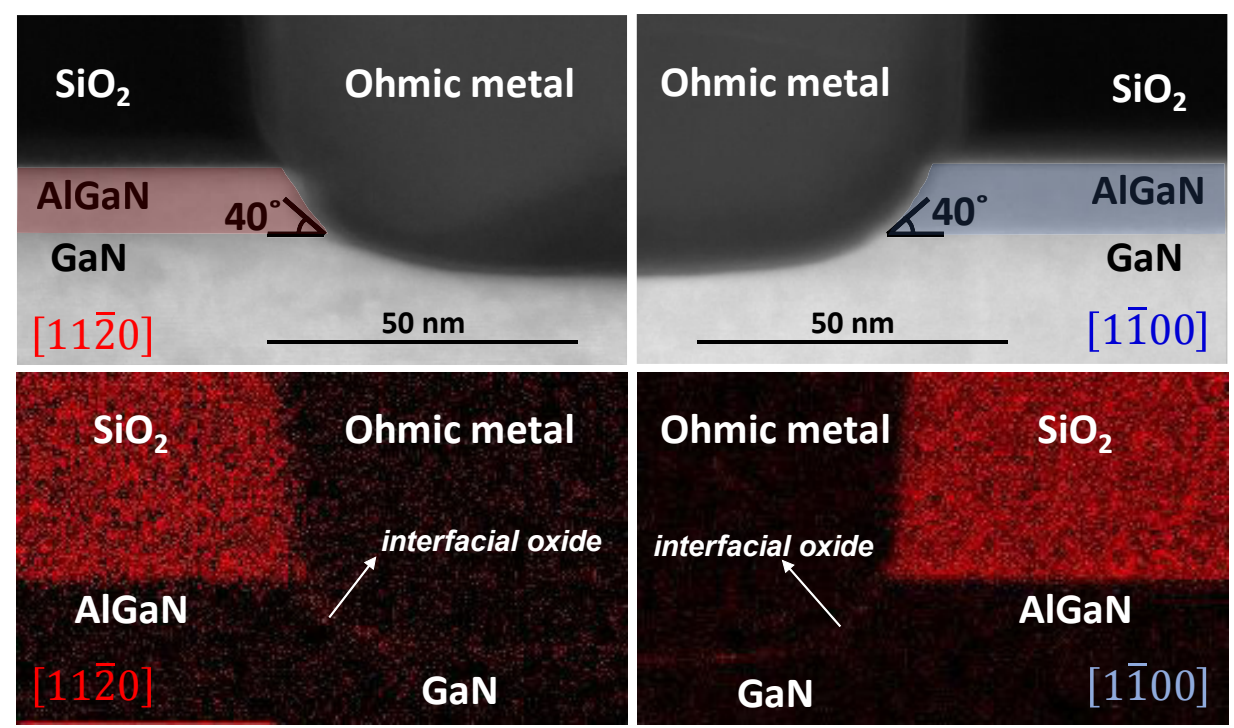

Fig. 5. (Color online) HAADF STEM cross-section micrograph (top) and STEM EDS oxygen mapping (bottom) of the ohmic contact area of transistors parallel to the [1120] direction or parallel to the [1ํㅣㄹ direction for the HCl-based wet cleaning before ohmic metal deposition.

contact resistance is expected for a more sloped sidewall. ${ }^{39)}$ We have compared the ohmic recess profiles for both crystal orientations by high angle annular dark field scanning transmission electron microscopy (HAADF STEM). The etch profiles are compared in Fig. 5 (top). For both directions, the ohmic recessing penetrates $\sim 6.5 \mathrm{~nm}$ in the $\mathrm{GaN}$ channel, and an identical sidewall slope of $\sim 40^{\circ}$ was measured for both crystal orientations. For this reason, it is unlikely that the ohmic dry recessing step is responsible for the observed difference in ohmic contact resistance.

In contrast to dry plasma etching, the GaN crystal is known to be highly anisotropic for wet chemical etching. It is known that both non-polar planes have a different atomic arrangement and are chemically not equivalent. The $a$-plane appears to be less chemically stable compared to the $m$-plane due to the higher surface energy. ${ }^{40)}$ This is translated in a higher wet etch rate for the $a$-plane. ${ }^{41,42)}$ Though more in depth investigation is needed, the lower contact resistance for the transistor parallel to the $a$-plane can be possibly explained by faster removal of etch residues in the $\mathrm{HCl}$ wet clean before the ohmic metal deposition. The presence of residual surface oxides, after dry etching, strip and the HCl-based wet cleaning step was probed by oxygen $\mathrm{K} \alpha$ energy dispersive $\mathrm{X}$ ray spectroscopy (EDS). Comparison of the oxygen map- pings for both directions (Fig. 5, bottom) shows the presence of residual oxygen at both the exposed $\mathrm{GaN}$ channel and the AlGaN barrier recessed sidewalls for both transistor orientations, but the technique unfortunately lacks sensitivity to be conclusive about possible differences in residual surface oxides between both transistor orientations.

The assumption of insufficient removal of the sidewall damage and/or etch residues after wet chemical cleaning before ohmic metal deposition, was verified by replacing the HCl-based clean by a more efficient sulfuric/ammonia peroxide (SPM/APM) cleaning mixture. In this case the ohmic contact resistance is as low as $0.3 \pm 0.02 \Omega \cdot \mathrm{mm}$ for both directions, resulting in low $R_{\mathrm{on}}$ values of $5.26 \pm$ $0.08 \Omega \cdot \mathrm{mm}$ for both transistor directions (Fig. 6).

\section{Conclusions}

For a non-optimized $\mathrm{HCl}$-based wet clean, recessed Au-free ohmic contacts in $\mathrm{p}-\mathrm{GaN}$ gate e-mode HEMTs rotated along the [1 $1 \overline{1} 00]$ direction result in lower contact resistance, and hereby higher transistor drive current and lower on-resistance with a tighter distribution over the $200 \mathrm{~mm}$ wafer. This is explained by more favorable etch and cleaning conditions when exposing the non-polar $a$-plane at the sidewalls of the ohmic recess, resulting in a larger process window for the 
ohmic contact process. The orientation-dependent contact resistance however disappears for a more efficient SPM/ APM-based wet clean prior to ohmic metal deposition, resulting in a low $R_{\mathrm{c}}$ value of $0.3 \Omega \cdot \mathrm{mm}$ independent of the crystal orientation.

1) S. Dimitrijev, J. Han, H. A. Moghadam, and A. Aminbeidokhti, MRS Bull. 40, 399 (2015)

2) O. Hilt, E. Bahat-Treidel, A. Knauer, F. Brunner, R. Zhytnytska, and J. Würfl, MRS Bull. 40, 418 (2015).

3) K. J. Chen, O. Häberlen, A. Lidow, C. L. Tsai, T. Ueda, Y. Uemoto, and Y. Wu, IEEE Trans. Electron Devices 64, 779 (2017).

4) Y. Uemoto, M. Hikita, H. Ueno, H. Matsuo, H. Ishida, M. Yanagihara, T. Ueda, T. Tanaka, and D. Ueda, IEEE Trans. Electron Devices 54, 3393 (2007).

5) T. Ueda, H. Handa, Y. Kinoshita, H. Umeda, S. Ujita, R. Kajitani, M. Ogawa, K. Tanaka, T. Morita, S. Tamura, H. Ishida, and M. Ishida, IEDM Tech. Dig., 2014, p. 283.

6) H. Yuliang, Z. Lian, C. Zhe, Z. Yun, A. Yujie, Z. Yongbing, L. Hongxi, W Junxi, and L. Jinmin, J. Semicond. 37, 114002 (2016)

7) D. Shibata, R. Kajitani, M. Ogawa, K. Tanaka, S. Tamura, T. Hatsuda, M. Ishida, and T. Ueda, IEDM Tech. Dig., 2016, p. 248.

8) K. Cheng, H. Liang, M. Van Hove, K. Geens, B. De Jaeger, P. Srivastava, X. Kang, P. Favia, H. Bender, S. Decoutere, J. Dekoster, J. I. del Agua Borniquel, S. W. Jun, and H. Chung, Appl. Phys. Express 5, 011002 (2012).

9) D. Marcon, B. De Jaeger, S. Halder, N. Vranckx, G. Mannaert, M. Van Hove, and S. Decoutere, IEEE Trans. Semicond. Manuf. 26, 361 (2013).

10) S. Lenci, B. De Jaeger, L. Carbonell, J. Hu, G. Mannaert, D. Wellekens, S You, B. Bakeroot, and S. Decoutere, IEEE Electron Device Lett. 34, 1035 (2013).

11) D. Marcon, M. Van Hove, B. De Jaeger, N. Posthuma, D. Wellekens, S. You, X. Kang, T.-L. Wu, M. Willems, S. Stoffels, and S. Decoutere, Proc. SPIE 9363, 936311 (2015).

12) D. Marcon, Y. N. Saripalli, and S. Decoutere, IEDM Tech. Dig., 2015, p. 414.

13) S. Stoffels, M. Zhao, R. Venegas, P. Kandaswamy, S. You, T. Novak, Y. Saripalli, M. Van Hove, and S. Decoutere, IEDM Tech. Dig., 2015, p. 911.

14) X. Li, M. Van Hove, M. Zhao, K. Geens, V.-P. Lempinen, J. Sormunen, G Groeseneken, and S. Decoutere, IEEE Electron Device Lett. 38, 918 (2017).

15) A. Malmros, H. Blanck, and N. Rorsman, Semicond. Sci. Technol. 26, 075006 (2011)

16) M. Van Hove, S. Boulay, S. R. Bahl, S. Stoffels, X. Kang, D. Wellekens, K Geens, A. Delabie, and S. Decoutere, IEEE Electron Device Lett. 33, 667 (2012)

17) G. Greco, F. Iucolano, and F. Roccaforte, Appl. Surf. Sci. 383, 324 (2016).

18) A. Shriki, R. Winter, Y. Calahorra, Y. Kauffmann, G. Ankonina, M. Eizenberg, and D. Ritter, J. Appl. Phys. 121, 065301 (2017).
19) A. Constant, J. Baele, P. Coppens, W. Qin, H. Ziad, E. De Backer, P. Moens, and M. Tack, J. Appl. Phys. 120, 104502 (2016).

20) D. Buttari, A. Chini, G. Meneghesso, E. Zanoni, B. Moran, S. Heikman, N. Q. Zhang, L. Shen, R. Coffie, S. P. DenBaars, and U. K. Mishra, IEEE Electron Device Lett. 23, 76 (2002).

21) M. Miyoshi, A. Imanishi, T. Egawa, H. Ishikawa, K. Asai, T. Shibata, M. Tanaka, and O. Oda, Jpn. J. Appl. Phys. 44, 6490 (2005).

22) W. S. Lau, J. B. H. Tan, and B. P. Singh, Microelectron. Reliab. 49, 558 (2009).

23) L. Wang, D.-H. Kim, and I. Adesida, Appl. Phys. Lett. 95, 172107 (2009).

24) S. Arulkumaran, N. G. Ing, V. Sahmuganathan, L. Zhihong, and B. Maung, Phys. Status Solidi C 7, 2412 (2010)

25) H.-S. Lee, D. S. Lee, and T. Palacios, IEEE Electron Device Lett. 32, 623 (2011).

26) B. De Jaeger, M. Van Hove, D. Wellekens, X. Kang, H. Liang, G. Mannaert, K. Geens, and S. Decoutere, Proc. Int. Symp. Power Semiconductor Devices and ICs, 2012, p. 49.

27) A. Firrincieli, B. De Jaeger, S. You, D. Wellekens, M. Van Hove, and S. Decoutere, Jpn. J. Appl. Phys. 53, 04EF01 (2014).

28) Z. Liu, M. Heuken, D. Fahle, G. I. Ng, and T. Palacios, Proc. Device Research Conf., 2014, p. 75

29) T.-L. Wu, D. Marcon, S. Stoffels, S. You, B. De Jaeger, M. Van Hove, G. Groeseneken, and S. Decoutere, Microelectron. Reliab. 54, 2232 (2014).

30) J. Bergsten, A. Malmros, M. Tordjman, P. Gamarra, C. Lacam, M.-A. di Forte-Poisson, and N. Rorsman, Semicond. Sci. Technol. 30, 105034 (2015).

31) J. Zhang, S. Huang, Q. Bao, X. Wang, K. Wei, Y. Zheng, Y. Li, C. Zhao, X Liu, Q. Zhou, W. Chen, and B. Zhang, Appl. Phys. Lett. 107, 262109 (2015).

32) H. Ishida, T. Murata, M. Ishii, Y. Hirose, Y. Uemoto, T. Tanaka, and D. Ueda, IEEE Trans. Electron Devices 53, 1524 (2006).

33) A. Konar, A. Verma, T. Fang, P. Zhao, R. Jana, and D. Jena, Semicond. Sci. Technol. 27, 024018 (2012).

34) N. E. Posthuma, S. You, H. Liang, N. Ronchi, X. Kang, D. Wellekens, Y. N. Saripalli, and S. Decoutere, Proc. Int. Symp. Power Semiconductor Devices and ICs, 2016, p. 95.

35) F. M. Mohammed, L. Wang, and I. Adesida, Appl. Phys. Lett. 88, 212107 (2006).

36) Y. Li, G. I. Ng, S. Arulkumaran, C. M. M. Kumar, K. S. Ang, M. J. Anand, H. Wang, R. Hofstetter, and G. Ye, Appl. Phys. Express 6, 116501 (2013).

37) M. Van Hove, N. Posthuma, K. Geens, D. Wellekens, and S. Decoutere, Ext. Abstr. Int. Conf. Solid State Devices and Materials, 2017, p. 647.

38) Z. Liu, Y. Wang, X. Xia, H. Yang, J. Li, and C. Gu, J. Vac. Sci. Technol. B 34, 041226 (2016)

39) S. Joglekar, M. Azize, M. Beeler, E. Monroy, and T. Palacios, Appl. Phys Lett. 109, 041602 (2016).

40) J. E. Northrup and J. Neugebauer, Phys. Rev. B 53, R10477 (1996).

41) D. A. Stocker, E. F. Schubert, and J. M. Redwing, Appl. Phys. Lett. 73, 2654 (1998).

42) G. Liu, B. Wen, T. Xie, A. Castillo, J.-Y. Ha, N. Sullivan, R. Debnath, A. Davydov, M. Peckerar, and A. Motayed, Microelectron. Eng. 142, 58 (2015) 